\title{
Turning Magnetic Density Separation into Green Business Using the Cyclic Innovation Model
}

\author{
E.J. Bakker*, A.J. Berkhout, L. Hartmann and P.C. Rem
}

Delft University of Technology, Faculty of Civil Engineering and Geosciences, Section Materials and Environment / Recycling Technology, Stevinweg 1, 2628 CN, The Netherlands

\begin{abstract}
The industrial implementation of Magnetic Density Separation (MDS) in the recycling of raw materials requires more than just the science of understanding why it works ('know-why') and the technology of how that can be accomplished ('know-how'). In addition, detailed knowledge of the market for streams of end-of-life products (supply side) and the market for recycled raw materials (demand side) are paramount to optimize the practical implementation and the design of an industrial plant. Indeed, in such a plant MDS is used to separate mixed streams of recycled materials into product streams which comply with market demands on grade and purity of the product compared with virgin streams. A recently developed non-linear innovation model is used to connect four fundamental cycles which continuously interact in the process of turning MDS technology into green business. The strength of this Cyclic Innovation Model lies in connecting technical capabilities with societal market needs. Only when a good match between the two is found a new technology such as, MDS develop into an innovation. The separation of polyolefins from end-of-life product is selected as one of the first industrial-sized applications of MDS in recycling. CIM is used to identify opportunities and challenges which need to be addressed to turn the MDS technology into an innovation that builds an economically attractive business in the context of a green society.
\end{abstract}

Keywords: Recycling, polyolefins, magnetic density separation, innovation, cyclic innovation model.

\section{INTRODUCTION}

Western economies use a lot of raw materials, many of which are getting more and scarcer. Rapidly growing demand from emerging economies of countries like China, India and Brazil increase the pressure on the availability of many essential commodities such as oil and various metals. The market for raw materials is growing and while demands rise it becomes increasingly more difficult to keep up the supply. This leads to soaring prices which temporarily slow down the demand. At the same time, higher prices make it economically more attractive to also mine raw materials that are more difficult to reach. For example, when the oil prices surged to a price level above $\$ 150$ per barrel, many small and difficult to utilize oil fields suddenly became viable to exploit. If that high price level would have persisted, new large-scale mining operations for even more difficult to reach oil reserves might have begun, for example the extraction of oil from the Athabasca oil sands (also known as tar sands) in Alberta, Canada.

The price of base metals has also increased dramatically over the past decade. The large demand for steel has caused a doubling of the price of iron ore from 2006 to 2008. The price of copper has increased from $\$ 1500$ per ton in 2002 to $\$ 9000 / t$ in 2008 . The worldwide economic crisis caused a steep price drop (less than $\$ 3000 / t$ in December 2008) as a result of a decrease in demand. However, presently (October

*Address correspondence to this author at the Delft University of Technology, Faculty of Civil Engineering and Geosciences, Section Materials and Environment / Recycling Technology, Stevinweg 1, $2628 \mathrm{CN}$, The Netherlands; Tel: +31 (0)1527 86040; E-mail: e.j.bakker@tudelft.nl
2009) the price level has risen again to $\$ 6000 /$ t. The same pattern is observed for other base metals as well. For example, aluminium traded at $\$ 1400$ per ton in 2002 , $\$ 3200 / \mathrm{t}$ in July 2008, $\$ 1300 / \mathrm{t}$ in February 2009 and is currently at $\$ 1800 / \mathrm{t}$. The price of virgin PP (copolymer, according to ICIS) from 2007 to 2009 is shown in Fig. (1).

Political and social forces for a 'greener' world are currently more prominent than ever before. The two foci for a responsible and sustainable society are: decreasing the use of raw materials and increasing the reuse (recycling) of end of life products. If we keep on mining ore for copper, nickel and many other essential metals, we will soon have depleted all but the hardest to reach ore deposits. This will introduce scarcity and consequently a price explosion. The clever way to proceed is as a sustainable society which closes cycles in the use of precious raw materials. This means that we need to increase the recycling of valuable raw materials from end of life products. Already, incinerated household waste (bottom ashes) is the richest source of copper (in terms of grade) on the planet. Bottom ashes contain a higher percentage (by weight) of copper than even the richest ore deposits from which we produce virgin copper. Moreover, the copper in bottom ashes is already in the metallic state, while copper ore is predominantly the mineral chalcopyrite $\left(\mathrm{CuFeS}_{2}\right)$ which requires expensive (in terms of energy use) and potentially polluting hydrometallurgical processes to produce metallic copper.

Recently, a new green movement has evolved in response to the publication of the book 'Cradle to Cradle' by William McDonough and Michael Braungart [1]. According to Lovins [2] Cradle to Cradle (or C2C) is "a holistic economic, industrial and social framework that seeks to create systems 


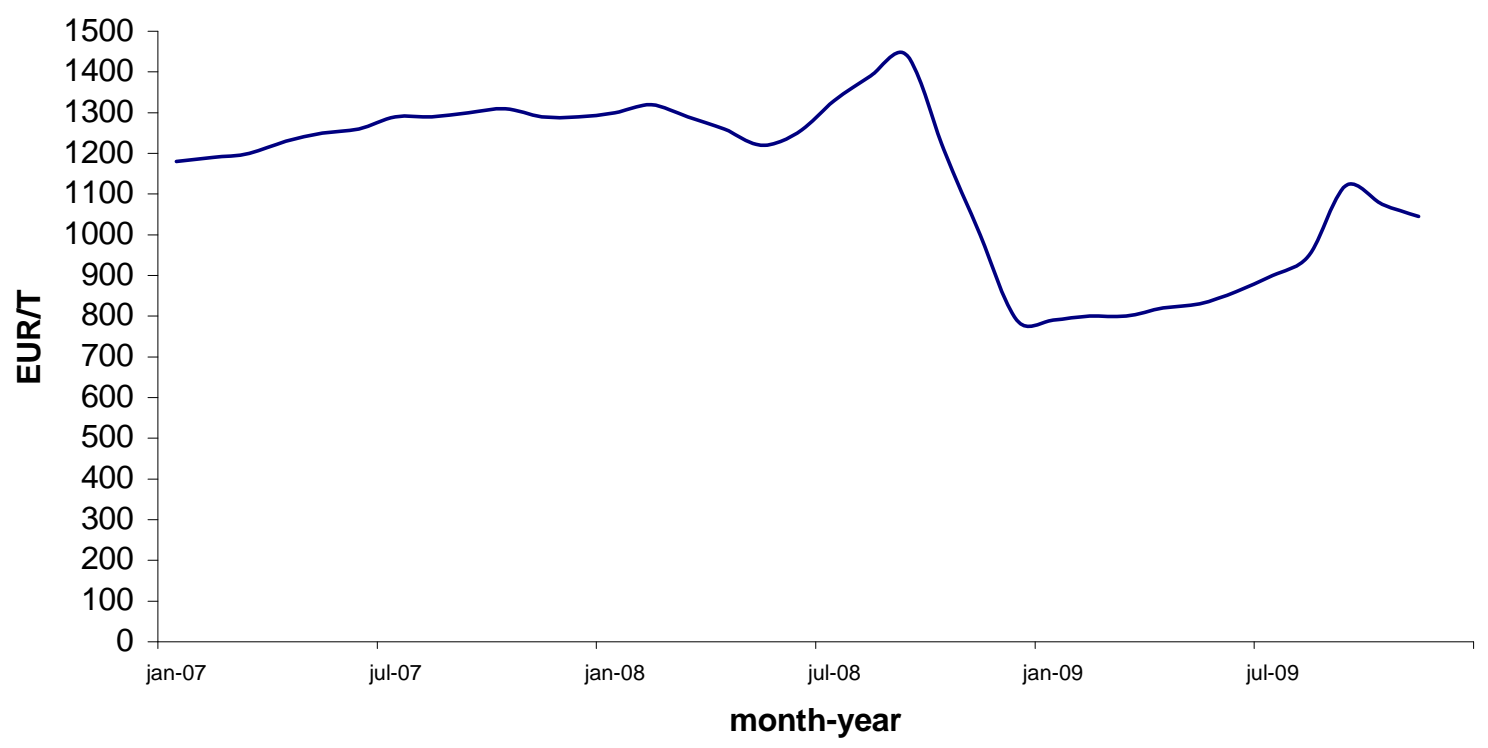

Fig. (1). The price per month of virgin PP (copolymer, according to ICIS) from 2007 to 2009.

that are not just efficient but essentially waste free". However, we think that a successful application of $\mathrm{C} 2 \mathrm{C}$ requires careful attention to the way in which we reuse our end of life products. The $\mathrm{C} 2 \mathrm{C}$ movement has attracted environmental activists insisting that not only should copper be recycled and used again as copper, but the same 'reuse as is' dogma should be applied always and everywhere. However, in many cases (such as the recycling of paper, wood and several polyolefins) this approach is questionable because it may cost more in terms of energy and other resources to reuse them - as they were used originally - than to use them in another (more environmentally responsible) manner. For example, while 'plastic-to-plastic' may sound appealing as a specific example of the cradle-to-cradle concept, it may require more resources than producing new (virgin) plastic from oil. If that is indeed the case, burning the end of life plastic to generate energy is probably the preferred option from an environmental point of view. Point solutions will inevitably generate disappointing results. Extensive Life Cycle Analyses (LCAs), showing the big picture, are required to decide which is the best approach.

Clearly there are many old and new challenges for both the mining and the recycling industry, both of which are suppliers of raw materials. New innovative technologies will play an important role in facing these challenges. Big fluctuations in the world market prices for raw materials introduce big uncertainties in the economic returns of recycling operations. This introduces a critical faceoff between the economic viability and the societal desirability of recycling, because the decision to build a recycling plant is generally determined by the market price of the output streams rather than by ecological arguments and sustainability considerations. When copper is cheap, it is too expensive to recycle and therefore it disappears in landfills at the end of life of the products that contain copper. But when the market price of copper is high, it becomes economically attractive to recycle copper from end of life products. But copper that previously disappeared into landfills is beyond recycling until the price of copper reaches such extreme heights that excavation of and subsequent recycling from landfills becomes viable. In this economically driven mechanism the market price determines whether or not a sustainable approach to the use of raw materials is chosen. In the new innovation concept we propose, technological renewal and market transitions are cyclically interconnected.

In this paper we introduce a new technology, Magnetic Density Separation (MDS) which can be used in the recycling (separation) of materials with different densities. Because it is fast, accurate and affordable it brings down the economic threshold above which recycling is cost efficient. Although MDS technology is very promising, innovation is more than just technology push. Economic, social, and cultural aspects are often decisive in determining a successful market introduction. The essence of innovation is to successfully bring new (systems of) product-service combinations to the market. No matter how creative the design or how clever the development behind a technological invention may be, it can never be classified as an innovation if the market introduction fails. Before the implementation of the full potential of MDS technology becomes reality, several important hurdles have to be taken on this transition path that connects the current situation with the image of the future. An advanced innovation concept is needed to turn this new technology into a profitable business.

A new conceptual framework, the Cyclic Innovation Model (CIM), for managing innovations has been developed at Delft University of Technology. CIM avoids disciplinary segmentation and takes the technological, economic, social, and cultural aspects of innovation into account at all stages along the innovation path. From CIM it becomes clear that the interaction between technical capabilities and marker forces determines the success or failure of an innovation 
project. The implementation of the MDS technology in CIM is discussed in this paper. It is argued that this concept helps to accelerate reaching the full potential of MDS technology in creating a better, more 'green' mining and recycling industry.

\section{MAGNETIC DENSITY SEPARATION}

The standard route of processing ore or waste to high grade materials is to first liberate the various materials that are present in the feed by crushing, milling, cutting or shredding. Then, the resulting flow of particles is classified with screening, into narrow size fractions to improve the final separation. Density separation is used on a large scale in the beneficiation of ore and waste. Methods that use solely the density differences of materials are successful because the separation is, in principle, not influenced by size or shape of the materials, resulting in high grade products. Unfortunately, conventional methods have limitations in the density that can be reached. The limit for heavy liquids is 4.9 $\times 10^{3} \mathrm{~kg} / \mathrm{m}^{3}$ [3] (Table 1). Heavy liquids, especially in the higher density ranges, are expensive, toxic or difficult to recover after separation. Besides the $4.9 \times 10^{3} \mathrm{~kg} / \mathrm{m}^{3}$ density limit, these liquids are not easily used on an industrial scale. Heavy liquids created with fine, heavy particles suspended in water can reach a density up to $5.5 \times 10^{3} \mathrm{~kg} / \mathrm{m}^{3}$ (Table 2). Magnetite or ferrosilicon is widely used in recycling of metals, to clean coal and ore concentration, because of the easiness to recover after separation of the particles (conventional magnetic separator). In this way, a density of about $3,0 \times 10^{3} \mathrm{~kg} / \mathrm{m}^{3}$ is available on an industrial scale. However, this so called Heavy Medium Separation (HMS), needs a high capacity to be economically feasible.

Table 1. Typical Liquids which are Used for Heavy Liquid Separation [3]

\begin{tabular}{|c|c|}
\hline Heavy Liquid & $\begin{array}{c}\text { Density at } 20^{\circ} \mathrm{C} \\
\left(\times 10^{3} \mathrm{~kg} / \mathrm{m}^{3}\right)\end{array}$ \\
\hline Trichlorethene & 1.46 \\
\hline Trichlorofluoromethane & 1.50 \\
\hline Tetrachloromethane (carbon tetrachloride) & 1.59 \\
\hline Tetrachloroethene (perchloroethylene) & 1.62 \\
\hline Pentachloroethane (pentalin) & 1.68 \\
\hline Bromochloromethane & 1.99 \\
\hline 1,1 Dibromoethane (ethylene dibromide) & 2.05 \\
\hline Dibromethane (methylene bromide) & 2.49 \\
\hline Polytungstates & 2.85 \\
\hline Tribromomethane (bromoform) & 2.89 \\
\hline 1,1,2,2 Tetrabromoethane (acetylene tetrabromide) & 2.97 \\
\hline Duomethane (methylene iodide) & 3.33 \\
\hline Clerici solution $\left(\mathrm{CHCO}_{2} \mathrm{TI}, \mathrm{C}_{3} \mathrm{H}_{2} \mathrm{O}_{4} \mathrm{TI}_{2}, \mathrm{H}_{2} \mathrm{O}\right)$ & 4.9 \\
\hline
\end{tabular}

One exception to the limitations of density is Ferrohydrostatic Separation (FHS) [4], which can attain densities higher than $10.0 \times 10^{3} \mathrm{~kg} / \mathrm{m}^{3}$. This is achieved by the combination of magnetic liquids and a gradient magnetic field. However, although this method has been known for almost fifty years, it is not implemented in industry on a large scale. FHS creates an artificial separation density that is higher than water, but homogeneous distributed throughout the medium. This results in a complex geometry of (electro) magnets. The magnetic field itself limits the capacity because the apparent separation density is not completely homogeneously distributed. Also, the geometry is too complex to be upscaled to industrial sizes.

Table 2. Densities of Several $(0.043-0.174 \mathrm{~mm})$ Suspensions with their Apparent Viscosity

\begin{tabular}{|c|c|c|}
\hline $\begin{array}{c}\text { Mineral/ } \\
\text { Element }\end{array}$ & $\begin{array}{c}\text { Density } \\
\left(\times \mathbf{1 0}^{\mathbf{3}} \mathbf{~ k g} / \mathbf{m}^{\mathbf{3}}\right)\end{array}$ & $\begin{array}{c}\text { Apparent Viscosity } \\
\left(\times \mathbf{1 0}^{-3} \mathbf{P a} \cdot \mathbf{s}\right)\end{array}$ \\
\hline \hline $\mathrm{SiO}_{2}$ & 1.7 & $\approx 62$ \\
\hline $\mathrm{Fe}_{3} \mathrm{O}_{4}$ & 2.7 & $\approx 76$ \\
\hline $\mathrm{FeS}$ & 3.45 & $\approx 74$ \\
\hline $\mathrm{PbS}$ & 3.75 & $\approx 86$ \\
\hline $\mathrm{Pb}$ & 5.5 & $\approx 64$ \\
\hline
\end{tabular}

A new approach based on the same principle as FHS has been researched and developed since 2004. Like FHS, Magnetic Density Separation (MDS) uses a combination of magnetic liquids and a gradient magnetic field. But instead of aiming to create a separation medium with one uniformly distributed density requiring a complex geometry of the (electro) magnets, the simplicity of the magnet geometry was leading. This approach led to a design which can be scaled up to industrial capacity. Because the medium has stratified layers of different density, multiple fractions can be separated in a single process step. The research that has been done over the last five years [5-7], shows that MDS technology overcomes major problems that hamper the industrial scale application of FHS. MDS has the capability to reach densities from $0.8 \times 10^{3} \mathrm{~kg} / \mathrm{m}^{3}$ up to $20.0 \times 10^{3}$ $\mathrm{kg} / \mathrm{m}^{3}$. The technology can be economically feasible with throughputs in a range from $0.1 \mathrm{t} / \mathrm{h}$ to $10 \mathrm{t} / \mathrm{h}$ (even higher) depending on the input material. MDS provides an innovative solution to some of the challenges that the mining and recycling industry is facing.

The basic principle of MDS is to use magnetic liquids as the separation medium. In the absence of a magnetic field these liquids have a material density $\rho$, which is comparable to that of water. But in a gradient magnetic field, the force on a volume of the liquid is the sum of the gravity and the magnetic force. The resulting apparent density varies exponentially with the vertical coordinate $z$ :

$$
\rho_{\text {apparent }}=\rho+\frac{2 \pi M B_{0}}{g w} e^{-2 \pi z / w}
$$

where $M$ is the magnetization of the magnetic liquid, $B_{0}$ is the strength of the magnetic induction at the surface of the magnet $(z=0), g$ is the acceleration of gravity $\left(9.81 \mathrm{~m} / \mathrm{s}^{2}\right)$ and $w$ is the wavelength of the field. Magnetic liquids will have an apparent density according to this formula in the field of a horizontal magnet consisting of a series of alternating magnetic poles. The MDS separator segregates the feed into stratified layers of different materials, with each material 


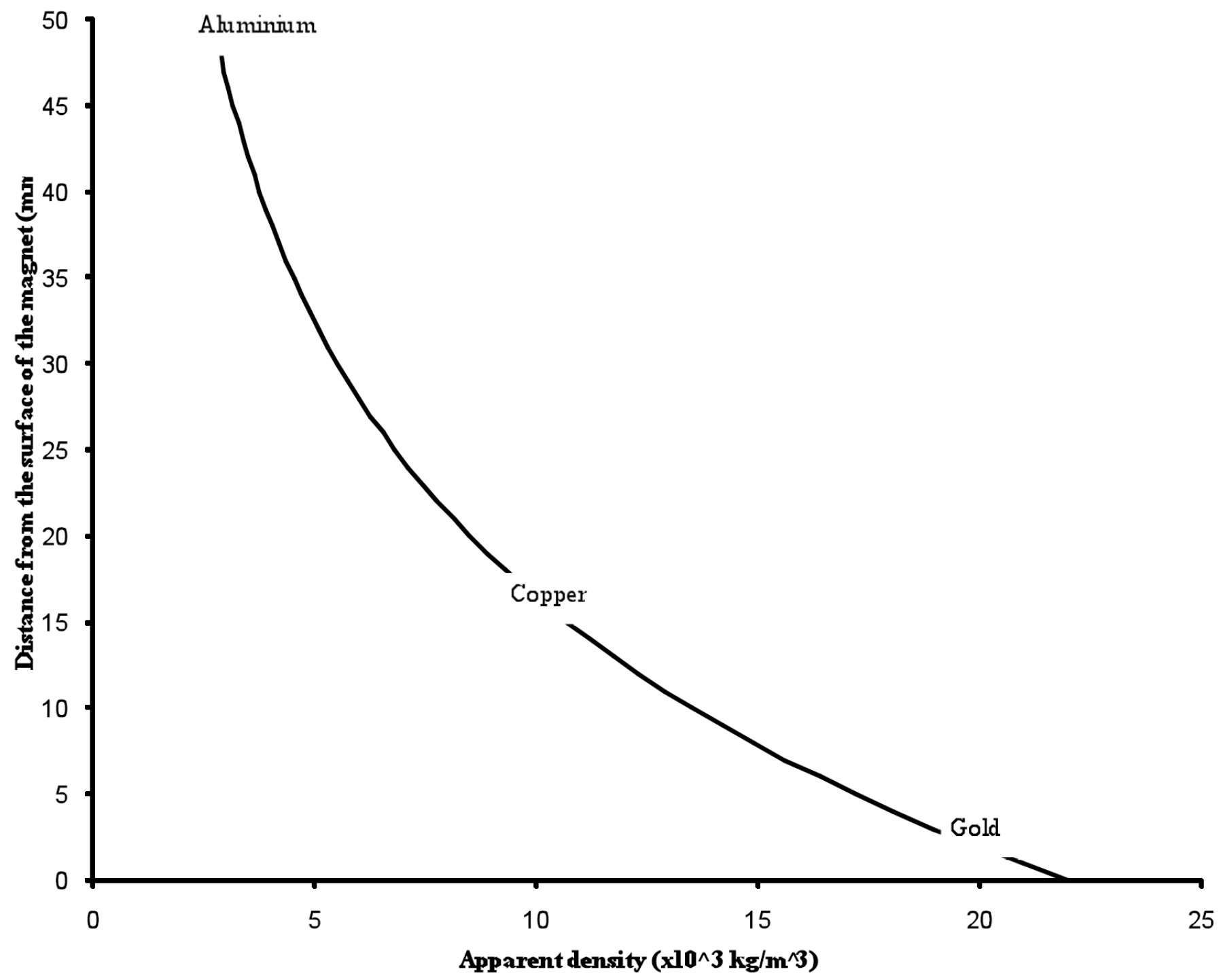

Fig. (2). Different apparent densities $\rho_{\text {apparent }}$ at a distance $\mathrm{z}(\mathrm{mm})$ from the surface of the magnet. In this case $\mathrm{M}=6500 \mathrm{~A} / \mathrm{m}, \rho=1174 \mathrm{~kg} / \mathrm{m}^{3}$, $\mathrm{B}_{0}=0.6 \mathrm{~T}$ and $\mathrm{w}=0.12 \mathrm{~m}$. Gold is floating at $3 \mathrm{~mm}$, Copper at $19 \mathrm{~mm}$ and Aluminium at $50 \mathrm{~mm}$.

floating at a specific distance above the magnet according to its density and the given formula. Fig. (2) shows an example where $M=6500 \mathrm{~A} / \mathrm{m}, \rho=1174 \mathrm{~kg} / \mathrm{m}^{3}, B_{0}=0.6 \mathrm{~T}$ and $w=0.12$ $\mathrm{m}$. Gold floats at $\mathrm{z}=3 \mathrm{~mm}$, Copper at $\mathrm{z}=19 \mathrm{~mm}$ and Aluminium at $z=50 \mathrm{~mm}$. Fig. (3) is a picture of particles floating at different heights. The yellow particles have the highest density and are closest to the magnet surface; The blue particles are the lightest and float at the largest distance from the magnet surface while the red particles are in between.

Fig. (4) shows the flow sheet of the MDS process applicable to any application. The first step is wetting. This is an important step because the separation is based on density. If the particles are not wetted properly, air attached to the particles will decrease the density. This wetting is especially important when the densities of the particles to be separated are close together, for example in the separation of the plastics PP and PE.

After wetting, the particles are separated in the MDS. There are two main MDS designs. The simplest one is functioning as a sink float system, which gives an output of two fractions (Fig. 5). With this MDS design with two fractions (MDS-TF), a conveyor belt is running on top of the magnet. The magnetic liquid flows over the conveyor belt in a direction perpendicular to the transport direction of the belt. Riffles on the surface of the belt, perpendicular on the direction of the liquid flow, have a specific height corresponding to a specific apparent density $\rho_{\text {cut }}$. The feed moves with the liquid flow and particles with a density $<\rho_{c u t}$ will flow with the liquid over the riffles. Particles with density $>\rho_{\text {cut }}$ will be blocked by the riffles. The ridges on the conveyer belt will transport these heavier particles in a direction perpendicular to the liquid flow.

However, it is also possible to separate the input stream into multiple fractions in a single process step (Fig. 6). With the MDS design with multiple fractions (MDS-MF), the magnetic liquid is flowing in a gutter on top of the magnet. The material is fed at a certain height within the liquid. The different particles will settle and subsequently float at their specific density heights from the surface of the magnet. At 


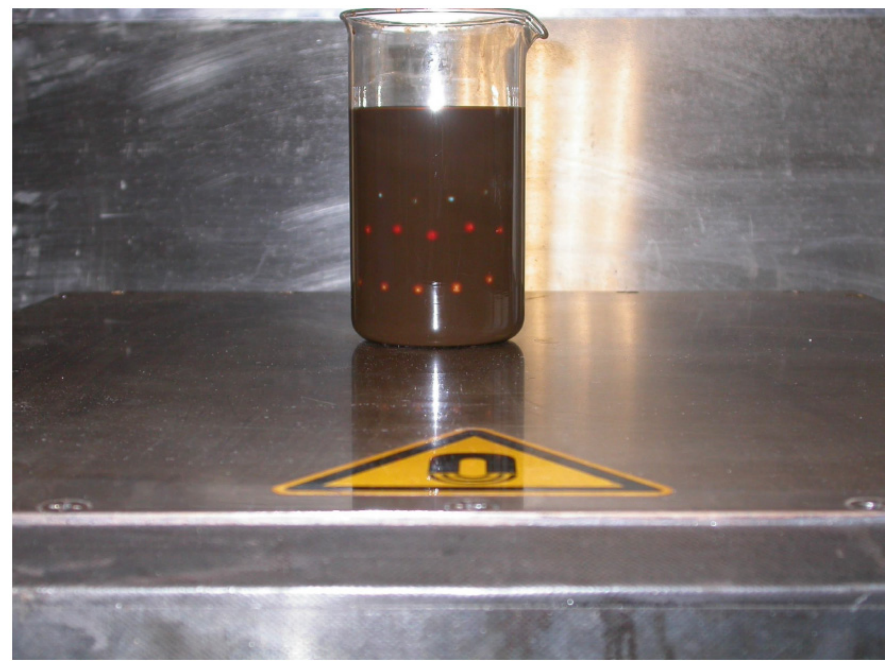

Fig. (3). Picture of plastic particles floating in a magnetic liquid. The liquid is placed in the magnetic field of the magnet which is perpendicular to the surface. This results in different apparent densities at different distances from the surface of the magnet. In this way the particles with the lowest density are floating at the highest position (blue particles). The particles with the highest density are the closest to the surface of the magnet (yellow particles), while the red particles are in between.

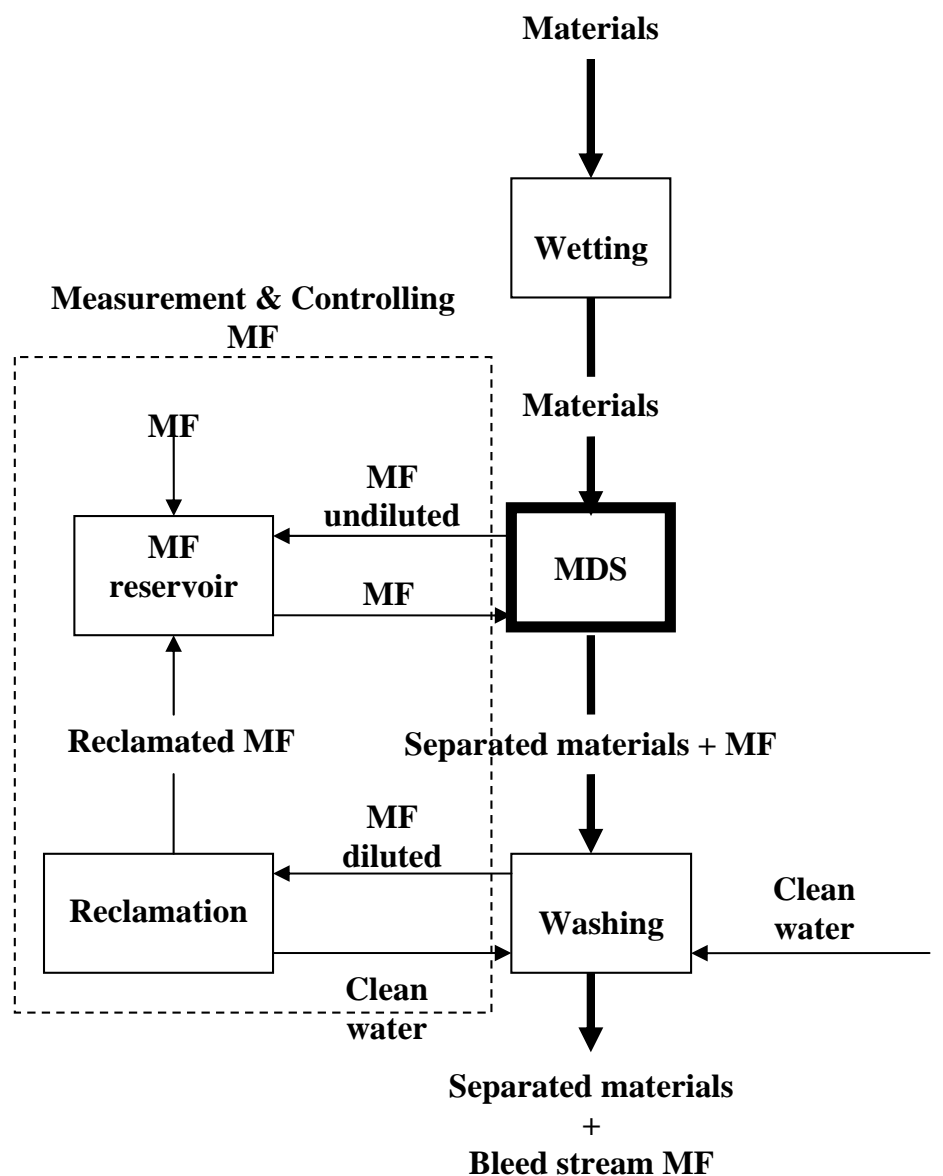

Fig. (4). Flow sheet of the MDS process. Wetting of the particles is essential in applications where the different densities are close together and the separation density is close to the density of water. After the separation, the particles need to be washed for reclamation of the liquid and/or for the quality of the product. The whole liquid cycle is measured and controlled.

the end of the gutter the different density fractions enter outlets at different heights and the particles are separated.
After the density separation, the output fractions are washed with clean water to remove the magnetic liquid 


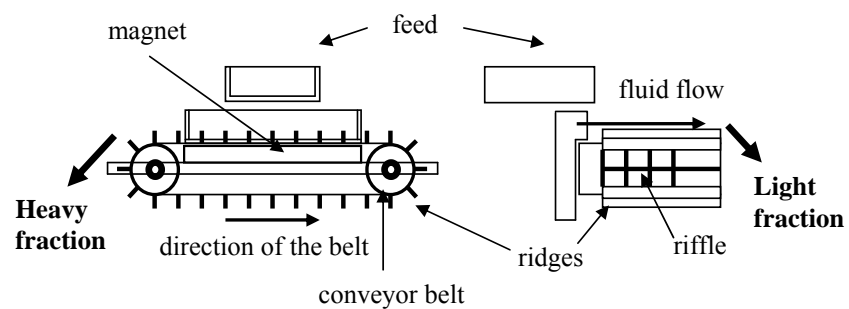

Fig. (5). Schematic drawing of the MDS-TF. The light fraction is going with the liquid flow, perpendicular to the direction of the conveyer belt. The heavier particles are transported in the direction of the conveyer belt.

adhered to the particles. There are two main reasons for washing. First, the magnetic liquid is too expensive to lose with the particles. By washing, the magnetic liquid from the particles, the liquid can be largely reclaimed. A bleed stream is needed to avoid a build up of fines and other contaminants. Second, the particles need to be cleaned for reuse. For example, PET bottles are predominantly transparent, so the recovered PET particles should also be transparent to be reused for bottle application.

The magnetic liquid is heavily diluted after the washing step. The additional water must be removed to reclaim the magnetic liquid. Research was done on the comparison of four different ways of reclaiming the magnetic liquid [8]: chemical precipitation, freeze crystallization, nanofiltration and evaporation.

\section{MDS RESEARCH}

Experimental results for four different applications of MDS are briefly discussed in this section. The first three (PET, copper/aluminium and precious metals) were carried out using MDS-TF. MDS-MF was used to separate a mixture of PP and PE.

\section{Removal of Contaminants from PET}

PET bottles are collected in Europe to be recycled. The PET is contaminated with PP, PE, aluminium, glass, stones and other materials. In today's practice, a lot of the contaminants are removed in a sequence of different separation units and the recovered PET can be reused in carpets, padding and clothes. However, there are still some contaminants particles present in the PET. MDS can remove these particles, thus generating a higher quality product while simplifying the process and reducing process costs.

Experiments with a MDS prototype were conducted, using PET flakes, artificially contaminated with aluminium particles [5]. The PET flakes had density of $1,350 \mathrm{~kg} / \mathrm{m}^{3}$. The PET particles used in the experiments were typically between 0.2 and $0.6 \mathrm{~mm}$ thick and with diameters from 2.5 $\mathrm{mm}$ to $20 \mathrm{~mm}$. The density of the aluminium particles was $2,700 \mathrm{~kg} / \mathrm{m}^{3}$. The sizes of the aluminium particles were between 1 and $2 \mathrm{~mm}$, between 2 and $4 \mathrm{~mm}$ and larger than 4 $\mathrm{mm}$, each fraction containing 100 particles. The cut-density to separate aluminium and PET should be around 1,600 $\mathrm{kg} / \mathrm{m}^{3}$. The cut-density can be influenced by the magnetization of the magnetic fluid. The magnetic fluid as received from the manufacturer had a magnetization of 13.3 $\mathrm{kA} / \mathrm{m}$. The experiments were conducted with a dilution of
11.1, 19.0 and 29.8. The throughput during the tests was 1.8 $\mathrm{t} /(\mathrm{h} \cdot \mathrm{m})$.

At all three dilutions there were no significant amounts of PET in the aluminium fraction. This suggests that the dilution of the magnetic fluid can be even higher. The time for the aluminium particles to reach the conveyor belt decreased with increasing dilution of the fluid. An increasing size of the aluminium particles also decreased the time for separation of the contaminants. The amount of aluminium particles in the PET fraction was not influenced by the dilution. These aluminium particles are probably trapped inside the PET layer independently of the dilution. The trapped particles were predominantly the smaller particles.

\section{Separation of Copper and Aluminium}

A Heavy Medium Separator (HMS) can be used to separate aluminium and copper. An HMS is a sink-float tank with a medium that has a density between the densities of the materials that have to be separated. For aluminium $(2,700$ $\left.\mathrm{kg} / \mathrm{m}^{3}\right)$ and copper $\left(8,900 \mathrm{~kg} / \mathrm{m}^{3}\right)$ the medium is a suspension of a mixture of fine magnetite $\left(\mathrm{Fe}_{3} \mathrm{O}_{4}\right)$ and ferrosilicon $(\mathrm{FeSi})$ in water. This separation method needs a high throughput $(>$ 50 ton/h) to be profitable. Many recycling companies have mixtures of aluminium and copper, but not in these large quantities. Performing the separation in-house increases the profit margin from the market value of the copper/aluminium mixture to the market value of the two separated metals. MDS is already economical feasible with a throughput of 5 ton $/ \mathrm{h}$. An experiment was done with a small-scale MDS prototype.

First, a $20 \mathrm{~kg}$ sample $\mathrm{Cu} / \mathrm{Al}$ mixture from shredded refrigerators was used. The majority of the particles were flat pieces with a thickness of a few millimetres and a maximum length of about $10 \mathrm{~cm}$. Some of the particles were tubes of about $1 \mathrm{~cm}$ in diameter. The dilution of the magnetic liquid was 5 and the throughput of the material was $3.6 \mathrm{t} /(\mathrm{h} \cdot \mathrm{m})$. The resulting grade of the aluminium was $99.1 \%$ with a recovery of $96.8 \%$. The grade of the copper was $63.6 \%$ with a recovery of $85.9 \%$.

Next, about 80 ton of a $\mathrm{Cu} / \mathrm{Al}$ mixture was processed. Samples of about $1.5 \mathrm{~kg}$ were taken to determine grade and recovery of the products. The particles of the samples were approximately spherical with a maximum diameter of $4 \mathrm{~mm}$. The throughput of the material during sampling was 1 $\mathrm{t} /(\mathrm{h} \cdot \mathrm{m})$. The dilution of the magnetic liquid was 7.8 . The resulting grade of the aluminium was $99.4 \%$ with a recovery 
Table 3. Overall Analysis of the Light and Heavy Products

\begin{tabular}{|c|c|c|c|c|c|}
\hline & $\begin{array}{c}\text { 2-6mm HNF } \\
\text { Grade } \%\end{array}$ & \multicolumn{2}{|c|}{ Light } & \multicolumn{2}{|c|}{ Heavy } \\
\hline Iron & 2.5 & 1.0 & 36 & 19.5 & 64 \\
\hline Zinc & 20.2 & 21.3 & 96 & 8.7 & 4 \\
\hline Silver & 0.21 & 0.20 & 86 & 0.364 & 14 \\
\hline Lead & 7.8 & 6.3 & 74 & 24.5 & 26 \\
\hline Gold & 0.007 & 0.002 & 30 & 0.058 & 70 \\
\hline Total & 100 & 100 & 91.7 & 100 & 8.3 \\
\hline
\end{tabular}

of $90.2 \%$. The grade of the copper was $98.8 \%$ and a recovery of $99.9 \%$

\section{Precious Metals Recovery from the Heavy Non-Ferrous 2-6 mm Fraction}

A heavy non-ferrous (HNF) 2-6 mm fraction is produced in a treatment process for bottom ashes from municipal solid waste incinerators (MSWI). This concentrate fraction constitutes between 0.2 and 0.4 mass percentage of the $0-40$ $\mathrm{mm}$ input bottom ash. More than half of the mass content is copper and this fraction also contains precious metals. These precious metals can be extracted from HNF concentrates by expensive metallurgical processes $(500$ and $1000 € / t)$ but it is therefore more economical to further concentrate the precious metals by means of physical separation before resorting to hydro- or pyrometallurgy.

A batch of $75 \mathrm{~kg}$ 2-6 mm HNF concentrate was separated with MDS at a cut-density of approximately $10,000 \mathrm{~kg} / \mathrm{m}^{3}$ [6]. The light and heavy products were analysed. The recovery value for gold was calculated on the basis of the gold mass of recovered gold particles and the known gold content of large smelts from the past. The overall analysis of the light and heavy products is given in Table 3 .

\section{Processing a Mixture of Polyolefin's into High Quality Products}

About $80 \%$ (mass) of the PP particles from shredder residue has a density lower than $910 \mathrm{~kg} / \mathrm{m}^{3}$. Almost $100 \%$ (mass) of the LD-PE and more than $98 \%$ (mass) of the HDPE have a density higher than $910 \mathrm{~kg} / \mathrm{m}^{3}$. A sink-float process with a medium that has a density of $910 \mathrm{~kg} / \mathrm{m}^{3}$ should give good results for the PP fraction. To get both a high grade PP fraction and a high grade PE fraction, it is necessary to remove the fraction between $910 \mathrm{~kg} / \mathrm{m}^{3}$ and 930 $\mathrm{kg} / \mathrm{m}^{3}$. Using a conventional sink-float process would require a separation in two steps. Another disadvantage is the medium itself. Organic liquids (e.g. short chain alcohols) are used to produce a medium with a density lower than the density of water. This introduces economical and environmental problems. The MDS overcomes both disadvantages.
An experimental MDS setup was used to separate PP from a PP-PE mixture [7]. A grade of $98.1 \%$ and a recovery of $72.4 \%$ for PP was achieved.

\section{SEPARATION OF PP AND PE MIXTURES}

We chose the PP/PE separation as the MDS application we want to implement on an industrial scale using the CIM $[8,9]$. All the above mentioned applications can be implemented using CIM, but the separation of PP and PE appears to be the most complex case and therefore the most challenging case for this paper.

In 2005 the worldwide production of plastics was 230 million ton in [10], of which 53.5 million ton was produced in Europe. Polyethylene production - linear low density polyethylene (LLDPE), low density polyethylene (LDPE), high density polyethylene (HDPE) - was about 12 million ton while polypropylene production was about 9 million ton. These figures show that plastics are a large market, comparable to steel in terms of volume $\left(\mathrm{m}^{3}\right)$ (Plastics Europe). Fig. (7) shows that plastics production is still growing rapidly.

There are three outlets for plastics waste: energy recovery, safe disposal and mechanical recycling (Fig. 8). Out of 22 million ton of post-consumer plastic waste in Europe in 2005, 53\% was disposed, $29 \%$ was used for energy recovery and $18 \%$ was recycled [10]. According to Directive 2004/12/EC on packaging and packaging waste, a recycling level of $22.5 \%$ should be achieved for plastics packaging by the end of 2008. In a few countries, like Germany (49\% recycling in 2002), special logistic systems have already achieved much higher levels, but in most countries a lot still needs to be done (UK, $17.9 \%$ in 2003 ; France, $15 \%$ in 2002). New, more cost-effective separation technology can provide an important incentive to increase recycling rates.

One of the major opportunities is the recycling of polyolefin waste. Polyolefin fractions are often end fractions resulting from the recycling of cars, waste from electric and electronic equipment (WEEE) and packaging waste. Sinkfloat with water as the medium is the most commonly used process that creates mixtures of PP and PE as a float fraction. 


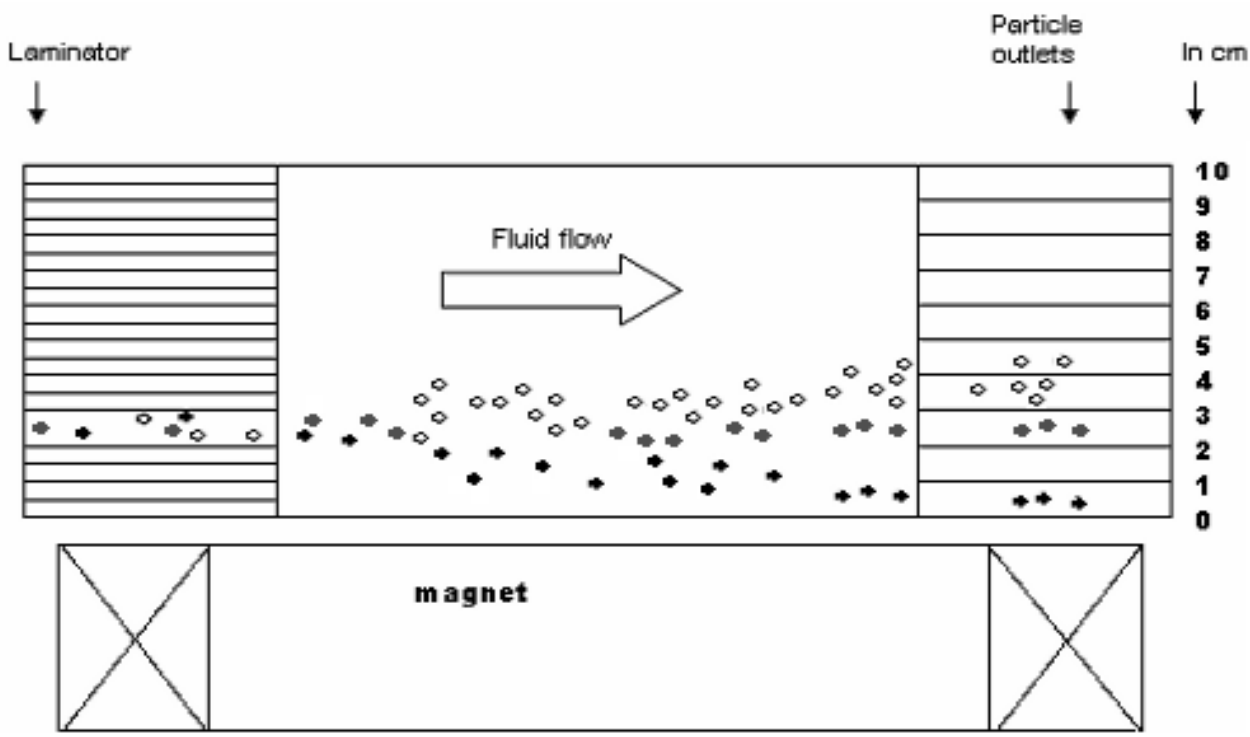

Fig. (6). Schematic drawing of the MDS-MF. The different density fractions are separated and transported towards the different density outlets.

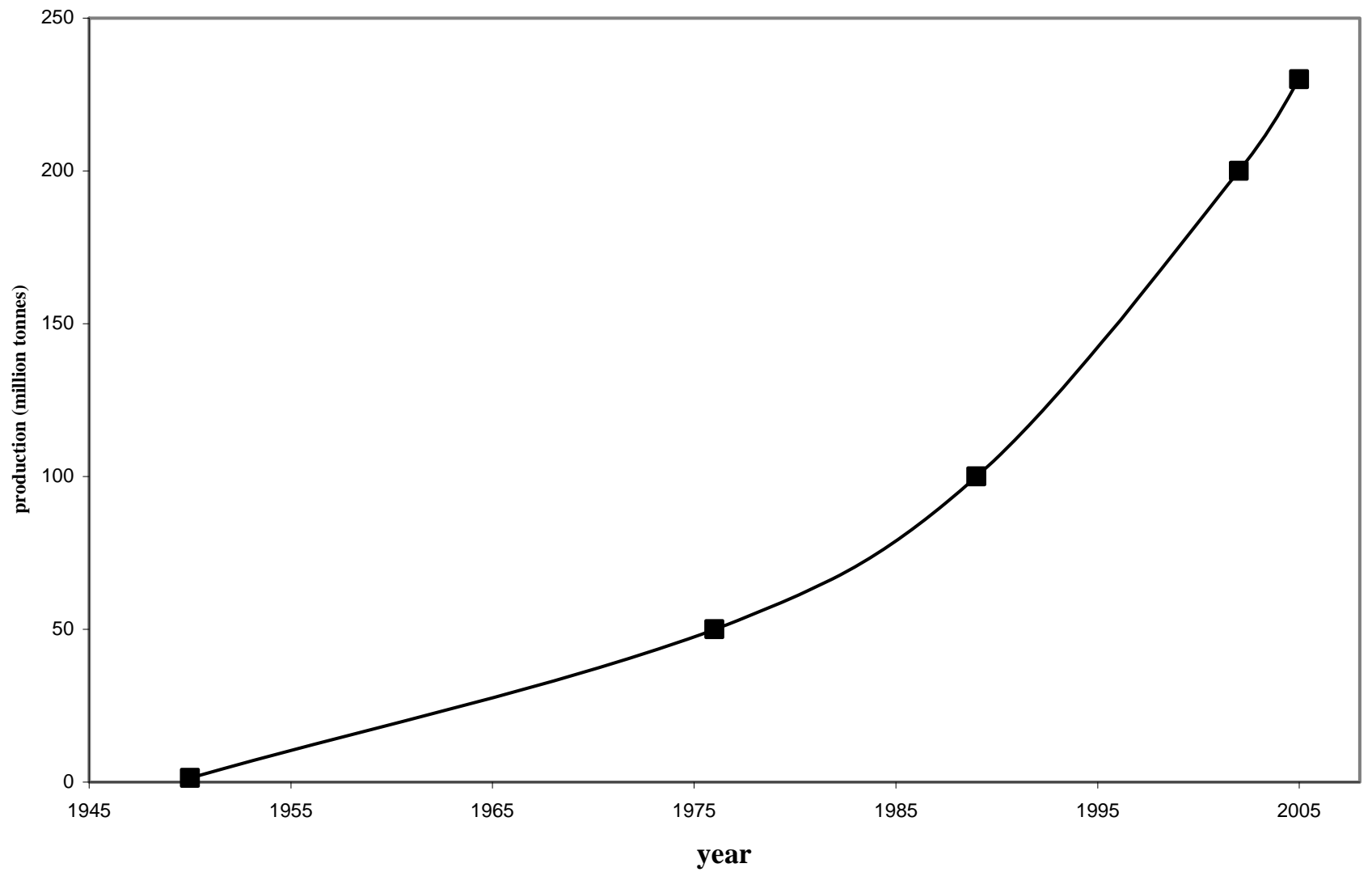

Fig. (7). Worldwide production of plastics in million ton from 1950 to 2005 (source Plastics Europe). This large plastic market is comparable to steel in terms of volume. Plastic production is still growing fast.

Typically, the PP:PE ratio ranges from 70:30 for car scrap to 25:75 for packaging waste. Such mixtures can be disposed in a safe way or used for energy recovery. A third option, reusing the mixture for consumer products, can only be possible after mechanical recycling. When reusing it as a high quality product the grade of PP and PE should be better than $97 \%$. Which of the three options is preferred should be determined by a Life Cycle Analysis. When opted for mechanical recycling, MDS is one of the promising techniques [11]. 


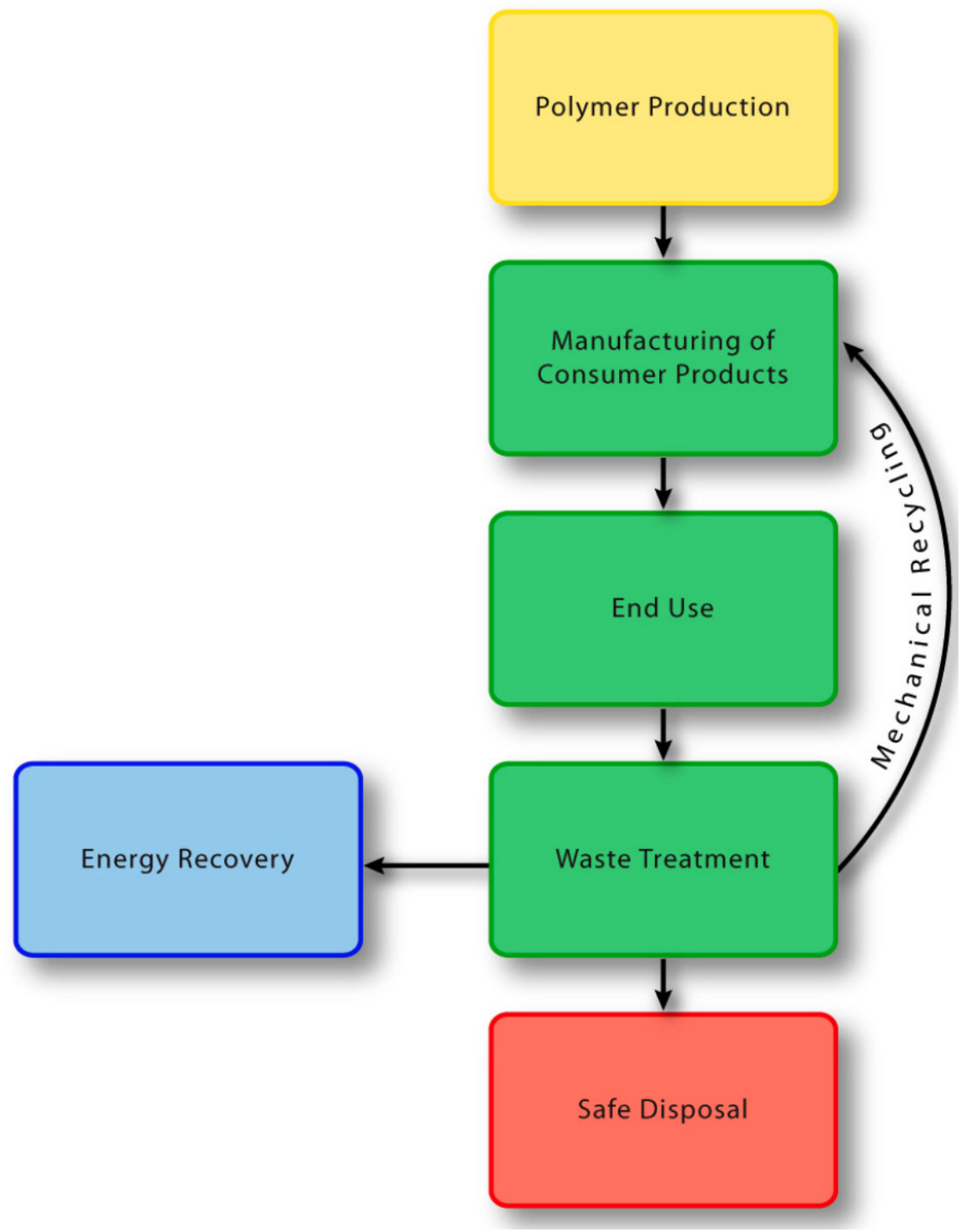

Fig. (8). Three outlets for end-of-life plastics: energy recovery, safe disposal and mechanical recycling. For every specific case, a complete Life Cycle Analysis should make clear which of those three options is the best one.

\section{THE CYCLIC INNOVATION MODEL (CIM)}

We propose to improve and accelerate the processes that lead to a green mining and recycling industry by making use of the latest insights in innovation. In the following we introduce the CIM and will demonstrate its potential for the MDS case. We start with discussing the most important limitations of traditional innovation models. Next, we describe the CIM which was developed on the basis of several decades of experience in the geo-energy industry. Finally, CIM is applied to MDS. Four sets of challenges in the fundamental cycles of CIM are identified, and a fifth set relates to the process model as a whole. These challenges need to be addressed to enable a successful market introduction. One of the distinguishing characteristics of CIM is that all these challenges are interrelated, meaning they cannot be addressed in isolation. In addition, they are best addressed in parallel, thus speeding up the innovation process.

\section{Limitations of Current Innovation Models}

Traditionally, innovation models are linear (explicit or implicit). They describe the processes along the innovation path as a causal sequence (much like in relay): investments in scientific research must lead to application-oriented development routes which subsequently ought to result in successful market introductions. If we invest enough in science and technology the rest will work out all right, that is often the reasoning (the innovation policy in the European Union aims at $R \& D$ budgets of the member states that amount to at least $3 \%$ of their GNP). Such a linear sciencepush approach in innovation policies is still taking place on a large scale, with the result that most innovation systems underperform.

Summarizing, the limitations of the current innovation models and schools are:

- Variations on linear thinking continue to dominate models of innovation. Actually, most innovation 
models show innovation paths, representing a stagegate type of activity and controlling the progress from idea to market introduction, rather than giving insight in the dynamics of actual innovation processes;

Science is viewed primarily as technology orientated (natural and life sciences) and R\&D is closely linked to manufacturing, causing insufficient attention to the social and behavioural sciences. As a consequence, the service component of innovation is hardly addressed;

The complex interactions between new technological capabilities and emerging markets are a vital part of the innovation process, but they are underexposed in current models;

- The role of the entrepreneur (individual or team) is not captured.

Box 1. change. Another example is on a micro scale, where we observe that the living cell - considered to be the most advanced chemical factory - is full of feedback. Molecular biologists believe that nature aims at minimum- energy systems and that minimum energy requires many feedback paths. If this is the case, we must conclude that our current innovation models, being characterized by little feedback, are squandering a lot of energy. Hence, innovation processes should not be forced into simple one-way pipelines but rather be organized by interconnected cycles with feed forward and feedback connections: from linear to nonlinear thinking. In that way, a dynamic network environment is created in which the soft sciences are linked to engineering, and where the hard sciences connect with market goals [9]. This is what is captured in the proposed innovation framework. Supported by today's powerful communication technology, serial process management along a linear path is replaced by parallel networking along a largely selforganizing circle. Vital decisions in innovation do not occur in the gates of a staged project management pipeline, but do occur at the innovation shop floor itself, or, in terms of our model at the nodes of the cyclic networks. It is our experience that young people like to work in such an environment.

\section{Double Dynamics Around Technological Renewal}

Fig. (9) shows two linked cycles, forming a double loop with technological renewal in a central position. The cyclic interaction processes for the development of new technologies take place in the hard sciences cycle (left-hand side of Fig. 9) with the help of a wide range of disciplines from the natural and life sciences. Technological renewal in this cycle is a cross-disciplinary activity: a team of scientists from different disciplines of the hard sciences is needed to develop a new technological capability (many-to-one relationship). In the last decades, we have seen that industrial firms have outsourced a large part of their science-based technological research to universities. Note that in Fig. (9) the hard sciences deal with quantitative models that not only explain the properties of physical systems ('know-why'), but are increasingly capable of predicting their behaviour as well. This predictability allows us to develop reliable technology with fully repeatable behaviour ('know-how').

Similarly, the cyclical interaction processes for the development of new products take place in the integrated engineering cycle (right-hand side of Fig. 9). Modern product development is a cross-technology process in which a package of different (often patented) technological capabilities is needed to design and prototype a new product (many-to-one relationship). As in cross-disciplinary science, here too we see that many different experts are needed to succeed. Nowadays, we observe that in most industrial firms specialized skills of technical suppliers from outside the firm play an important role in making the engineering process successful. This is consistent with the open innovation concept [13]. Note that 'products' refer to everything mankind builds: not only tangible products like houses, cars and computers, but also non-tangible products like websites, games, insurance policies, agreements and laws. And, combinations thereof, this means that engineering should integrate hard and soft components. 


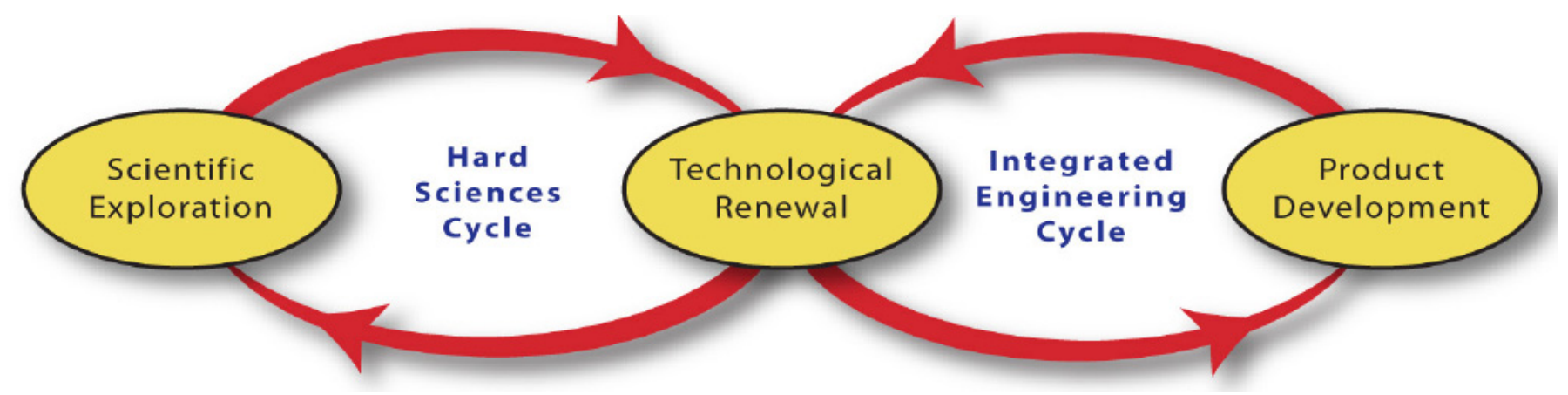

Fig. (9). The dynamics surrounding technological renewal are driven by the cyclical interaction between new discoveries in the hard sciences (left-hand side) as well as technical specifications for new process-product combinations (right-hand side).

Fig. (9) visualizes that in the hard sciences cycle, technological renewal is driven by new scientific insights: science push. It also shows that in the engineering cycle technological renewal is driven by new functional requirements in product development: function pull. The dynamics in technological change are therefore driven by new scientific insights as well as new product specifications. In a dynamic technological infrastructure, scientists and engineers must constantly inspire each other. To achieve this, research must be organized in a different manner: no barriers between the two cycles. In Fig. (8), the technological node should function as a knowledge-driven roundabout.

\section{Double Dynamics Around Market Transitions}

Fig. (10) also shows two linked cycles. In this case it is the world of market change rather than the world of technological change that plays the central role. The cyclical interaction processes for the development of new insights into emerging changes in demand - causing rising and falling markets - take place in the soft sciences cycle (lefthand side of Fig. (10) with the help of a wide range of different disciplines from the behavioural and social sciences. With these insights, new socio-technical solutions can be developed faster and with less economic risk. Understanding changes in demand is very much a crossdisciplinary activity: a team of disciplinary experts from the soft sciences is needed to assess and foresight shifts in societal needs and emotions as well as changes in trade conditions and regulations (many-to-one relationship). We see in all industrial sectors an increasing interest for this type of research, meaning a shift toward a more scientific approach to market studies. Note that in Fig. (10) the soft sciences deal with socio-economic models to explain the properties of markets and the underlying behaviour of consumers. Until today, the predictive power of these models needs improvement.

Likewise, the cyclic interaction processes required to serve the changing society with new product-service combinations take place in the differentiated services cycle (right-hand side of Fig. 10). In this cycle, services are seen as an invaluable link between products and markets: the combination of products and services determines customer value. Users play an increasing role in making the innovation process successful. Utilizing the creative input of customers is known as democratizing innovation [14]. It is interesting to note that in recent years the services sector has expanded considerably, not only because of the greater demand for services from the end-user but also because industry has outsourced many of its non-core processes. This trend is still going on, and will play an indispensable role in the forthcoming clean-tech era.

For many years the innovation literature overlooked the concept of new service innovation. Innovation was deemed to require a new physical "thing". We already argued that products are much more than tangible entities and, therefore, technology is much broader than the knowledge to design and build tangibles. In addition, the world of business suggested that new services could deliver potentially even more significant changes than new products. Together, they served as the kernel of new business models. The one caveat here is that frequently the new service is underpinned by a new technology application. This suggests that, today, interaction between the processes in Figs. $(\mathbf{9}, \mathbf{1 0})$ is at the base of most innovations.

\section{Cyclic Innovation Model (CIM)}

If we compare Figs. $(\mathbf{9}, \mathbf{1 0})$, the dual nature of scientific exploration and product development becomes clear: science has both hard and soft aspects, and product development has both technical and social aspects. Fig. (11) combines Figs. 9, 10). The result is the CIM $[9,15]$, a cross-disciplinary view of change processes (and their interactions) as they take place in an open innovation arena. Behavioural sciences and engineering as well as natural sciences and markets are brought together in a coherent system of synergetic processes with four principal nodes that function as roundabouts. The combination of the involved changes leads to a wealth of business opportunities. Here, entrepreneurship plays a central role: making use of those opportunities. The message is that without the drive of entrepreneurs there is no innovation, without innovation there is no new business, and without a new business there if no economic growth.

The most important feature of Fig. (11) is that the model architecture is not a chain but a circle: innovations build on innovations. Ideas create new concepts, successes create new challenges, and failures create new insights. Note that new ideas may start anywhere in the circle, causing a wave that propagates clockwise and anti-clockwise through the circle. In an innovative society partnerships are abundant and the speed of propagation along the circle is high, resulting in minimum travel time along the innovation path. Today, time is a crucial factor in innovation. 


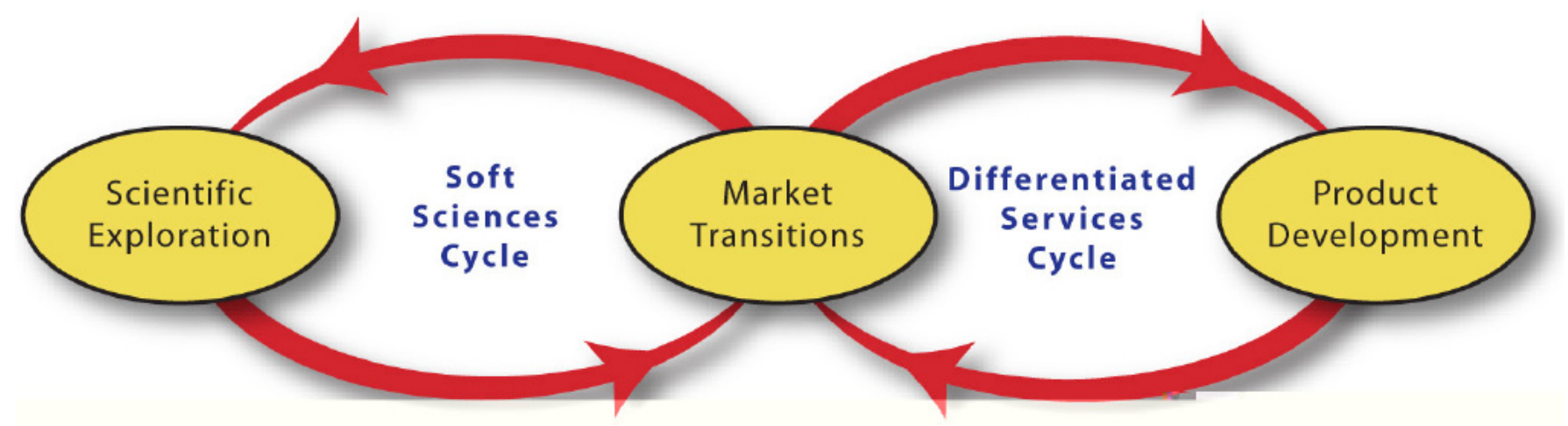

Fig. (10). The dynamics surrounding market transitions are driven by the cyclical interaction between new scientific insights in changing consumer behaviour (left-hand side) as well as new industrial supply of product-service combinations (right-hand side).

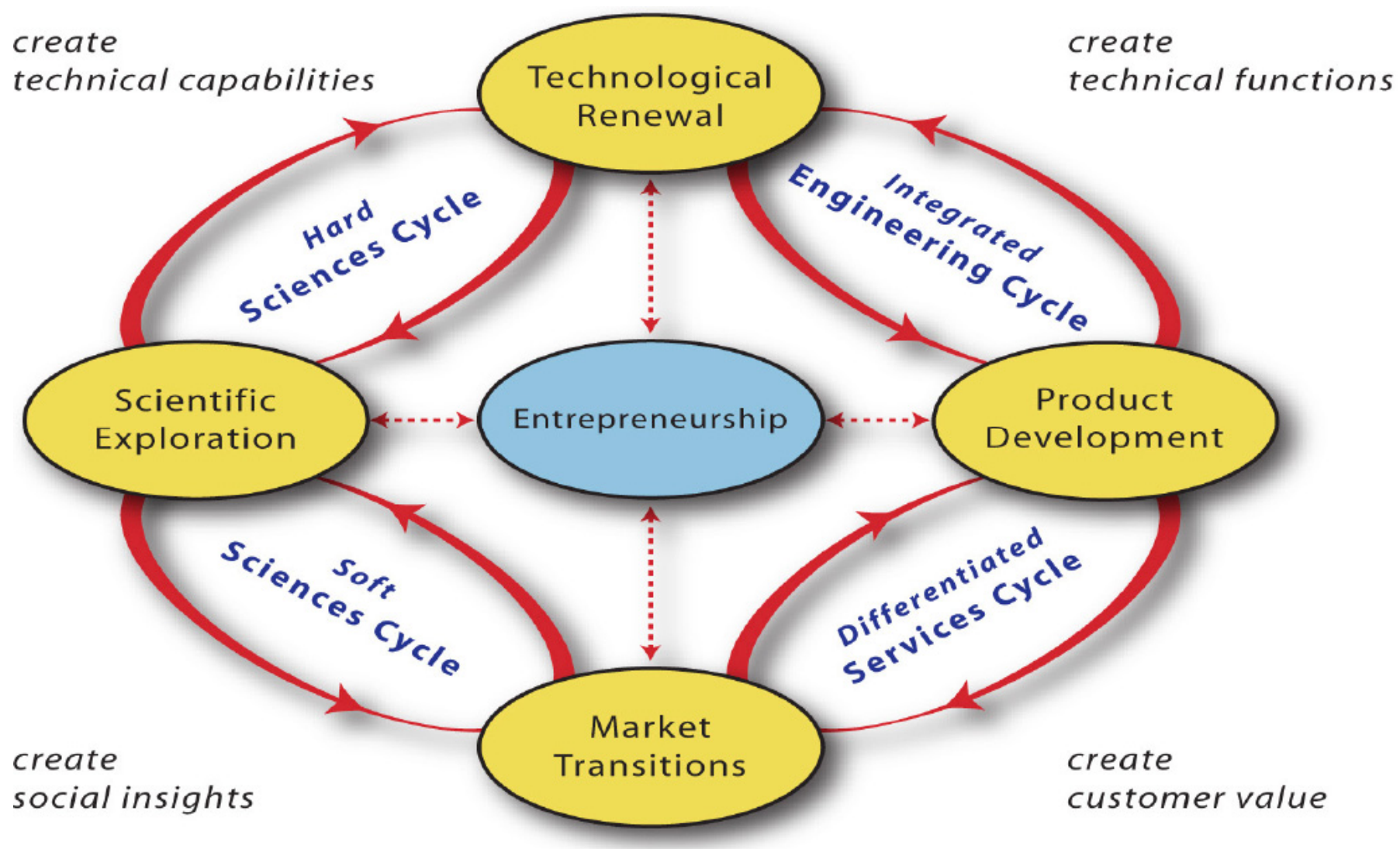

Fig. (11). The Cyclic Innovation Model (CIM) presents the processes in innovation by a circle of change. Changes in science (left) and industry (right), and changes in technology (top) and markets (bottom) are cyclically interconnected. Nodes function as roundabouts. Entrepreneurs function as circle captains.

Fig. (11) also shows that the proposed model portrays a system of dynamic processes - circle of change - with four "nodes of change": scientific exploration, technological renewal, product development, and market transitions. But more importantly, between these nodes there are "cycles of change" by which the dynamic processes in the nodes influence each other. In other words, they inspire, correct, and supplement each other (first-order dependency). This produces a system of linked cycles, which in turn also influence each other (higher-order dependencies). The result is a more or less synchronized regime of highly non-linear dynamic processes that spark a creative interaction between changes in science (left-hand side) and industry (right-hand side), and between changes in technology (top) and market (bottom). These are the dynamics that we have realized in the Delphi consortium. And, these are also the dynamics referred to by Richard Florida [16]. Note that in this type of self-organizing complexity, causality is not a meaningful concept anymore. Borrowing an observation from the famous Austrian physicist Wolfgang Pauli [17], many processes interact and we can no longer distinguish between cause and effect. Innovation resides in the world of selforganized chaos, steered by the ambitions of the firm. At a lower level, CIM shows that each cycle consists of a network with a high degree of self-organisation. 
Autonomous societal transitions manifest themselves in markets as changes in the need for products and services (the demand). Think of the huge influence of education and emancipation on a society. On the other hand, autonomous technological developments generate new products and services (the supply). Think of the huge influence of mobile and web-based communication technology on society. It is the cyclic interaction of both autonomous innovation drivers, social and technical, that will create new business with a maximum value for society. In that respect, specialized versions of the model can be formulated, depending on which values we particularly aim for. For instance, if we would like to emphasize changes in today's energy system aiming at a decentralized green alternative - then "market transitions" should be replaced by "energy transitions" in Fig. (11). Similarly, if we would like to emphasize changes in the global ecological system - aiming at maintaining biological diversity on our planet - then "market transitions" should be replaced by "ecological transitions". For the coming decades, quality of life will become one of the biggest drivers in innovation worldwide. This means that the transition node in the cyclic process model should be focused on the changing values in society at large: "societal transitions". We believe that the traditional single-value business models, aiming only at the financial returns on investment, should be transformed into models that are focussed on a multi-value proposition (business model innovation).

Box 2.

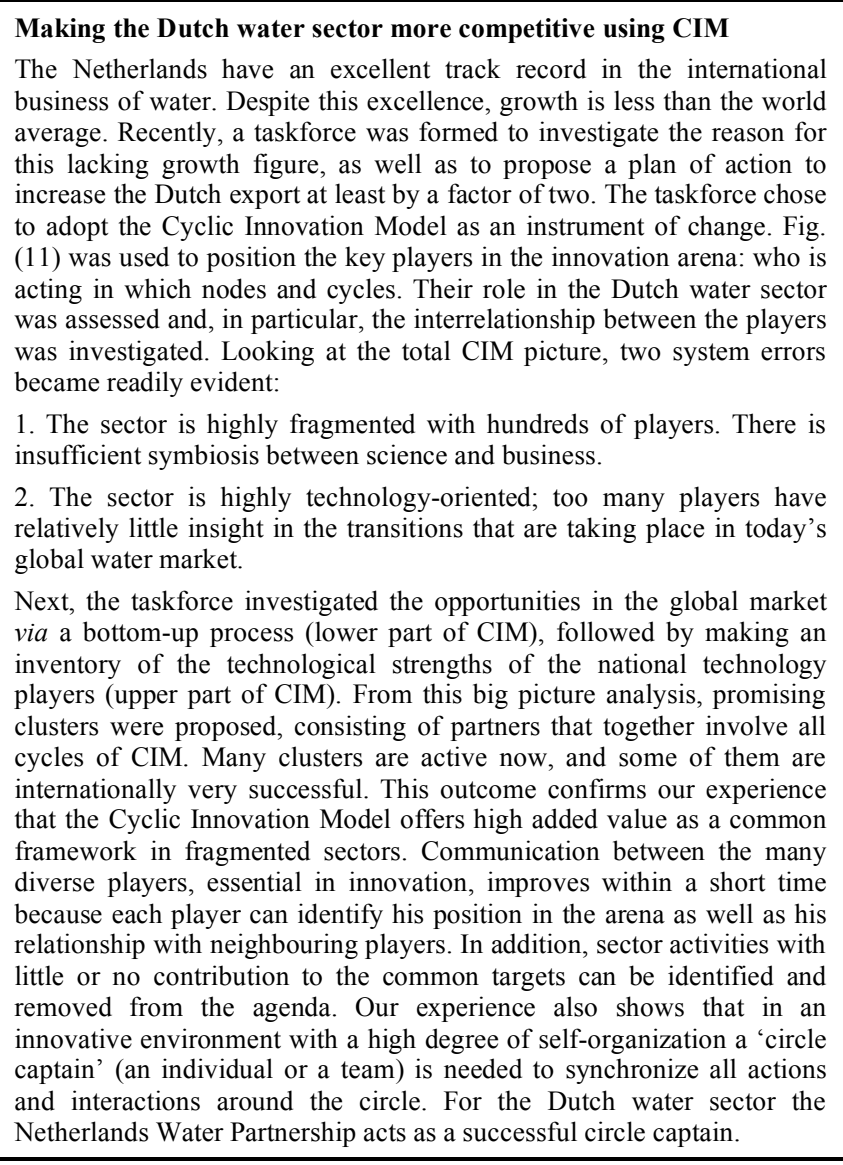

\section{The Role of Science in Innovation}

Fig. (11) shows that the left-hand side of the innovation circle is directed to research activities of the science community while the right-hand side addresses the innovation activities of the business community. In a productive innovation system, science and business will challenge each other continuously on technology-related (upper part) and market-related (lower part) issues. Looking at the upper part of the circle first, interesting examples of new enabling technology can be found in the bio-sciences where molecular biology has a great impact on innovations in the food industry. Crops, for instance, are made more resistant to diseases and pests, as well as increasing their yield and their nutritional value. It may be the solution to the insufficient security of the food supply on our planet. But bio-sciences also have great impact on the future of drugs, moving from one medicine for everybody to dna-related differentiation. Other impressive examples of new enabling technology can be found in the nanosciences where nanoparticles are designed to create coatings and materials with properties beyond imagination. Today, we already see spectacular applications in the pharmaceutical industry (drug delivery), but radical innovations may also be expected in the renewable energy sector (e.g., improved recycling capabilities). All these new technological capabilities will have a major impact on the way we live and, therefore, also on the way markets will evolve.

The above hard and soft sciences, however, also play another important role in innovation. Industrial $R \& D$ solutions are often discovered without fully understanding all aspects. Today, these types of industrial 'shortcuts' are important because, time has become a key issue in innovation. Refining the solution requires filling in the knowledge gaps, and here the science community becomes an important strategic partner: the explication of implicit knowledge. Instead of challenging scientists by asking 'surprise me', the industry is now asking scientists 'help me'. Note that this is a different role: science push is replaced by business pull. The cyclic innovation model shows that both roles of science in innovation should occur in a parallel manner and, even more importantly, they should be cyclically interconnected.

Let us now move to the business side of the model, being the right-hand part of the circle. After the development phase, technologies enter the phase of deployment [18]. For this phase, the upper left-hand side of the model plays a modest role; it is all about using these technologies to create new products and services for existing and emerging markets. Information and communication technology (ICT) is a typical example. With existing ICT platforms innovation primarily occurs in the engineering and services cycle. Examples are the emerging product-service combinations based on RFID ('tagging'), broadband communication (think of telecare) and new services facilitated by web 2.0 (and soon web 3.0). All these innovations are changing society (and the related markets) in a significant manner, but here the hard sciences are not in the driver seat. The soft components cannot be ignored.

Indeed, we see in the deployment phase an increasing impact of the soft sciences, bringing us to the lower left-hand side of the circle. Today, consumers are generally well- 
educated and they communicate with each other on products and services via social networks. Traditional marketing tricks do not work anymore. Firms must collect reliable information about customer needs and emotions in society. This is not only a matter of statistics; it is particularly a matter of the scientific knowledge behind statistics. The same applies for governments: effective regulations cannot be built without understanding the behaviour of their citizens. And the same also applies to the emotions in society with respect to sustainability. Today, there is an increasing pressure on the ecological and social footprint of future production systems. This type of research is an essential component in the transition to a fully sustainable economy. It also resides in the lower left-hand side of the model; social and behavioural scientists are the key players in such research. CIM shows that all these soft aspects (should) have again influence on decisions that are made in the technical world.

In conclusion, science plays a role in creating new technologies and assisting business to utilize these technologies in the early phase of application (cyclic interaction in the upper part of the circle). In the deployment phase of technologies, it is primarily a matter of the industry (cyclic interaction at the right-hand side of the circle); here, the role of scientists in the upper left-hand side of CIM is (and should be) small. In the deployment phase, however, major changes may occur in society. These changes should be identified and assessed by scientists active in the lower left-hand side of the innovation circle. Their role should be large.

Finally, the transformation to a sustainable society may be the biggest challenge mankind is facing. It requires changes in technology as well as in behaviour. The hard and soft sciences should work together with industry leaders to make this transformation happen. In terms of CIM, moving to a sustainable society requires synergy of activities around the entire innovation circle.

\section{Symbiosis}

From the above it follows that the innovation circle acts as a socio-technical framework that gives insight into the heart of the innovation process by asking the relevant questions, such as: What needs to be done where? Who are the collaborating parties? Where are they active in the circle? Is there a balance in investments between the different parts of the circle? Nobel laureate Robert Lucas points out that exchange of ideas is the principal driver for innovation [19]. So, in terms of the innovation circle (Fig. 11), the key question is whether there exists sufficient interaction around the circle. Particularly, for disruptive innovations an environment must be created where a large diversity of people with a broad range of backgrounds can freely interact, discuss ideas, and exchange information. This type of environment requires a significant change in the current institutional cultures and social structures, as disciplinary boundaries are deeply rooted in our organizations and solutions are often a collection of segmented optimizations.

We expect that synergetic alliances and complementary partnerships will empower the innovation processes along the innovation circle far beyond what we see today.

\section{USING CIM TO TURN MDS OF PP AND PE INTO PROFITABLE BUSINESS}

The cyclic innovation model can be applied to facilitate the implementation and adaption of new technologies with the potential to turn them into a profitable green business. A strategy has to be designed to move along the transition path from the current situation (today) to the image of the future (when the new technology is used) with minimum delay. There will be a number of obstacles along this transition path that must be overcome to realize a fast implementation. These obstacles must be identified and translated into challenges that need to be addressed. In many cases, these obstacles include a lack of innovation drivers and the use of linear concepts in innovation management. Chesbrough [13] shows that the well-known in-house, stage-gate model - a pipeline where promising ideas are developed toward successful market introductions - can be enhanced to a more open version, that allows external interactions from outside the pipeline. Chesbrough's extended pipeline was once more extended by Kirschbaum [20] by introducing the possibility of spin-in and spin-out. In reality, however, we observe that successful innovation processes are not a matter of simple one-way pipelines but rather of interconnected cycles with feed forward and feedback connections: from linear to nonlinear thinking. In that way, a net-centric environment is created in which the soft sciences are linked to engineering, and where the hard sciences connect with valorization goals. The links, which go forwards and backwards (cyclic processes), are an essential feature of dynamic systems [21, 22]. This is what you see in the innovation circle (Fig. 11), and that is why we use CIM in green innovation projects like MDS.

\section{MDS is a Class 4 Innovation}

In the context of the CIM (Fig. 11), we distinguish four classes of innovations. Class 1 innovations are based on changes in only one cycle (connecting two nodes). For instance, a new marketing concept for an existing productservice combination is a class 1 innovation. Similarly, class 2 innovations are based on changes in two cycles, etc. The most disruptive innovations are class 4 innovations in which all four cycles are simultaneously involved. This is the case for MDS. To manage the complex innovation process, challenges in each of the four cycles must be addressed. This leads to four sets of challenges: Challenges in the hard sciences cycle, challenges in the engineering cycle, challenges in the differentiated services cycle, and challenges in the soft sciences cycle. Finally, we have an indispensable set of challenges in the process model as a whole which have to be met by the entrepreneur (the circle captain) at the heart of the innovation processes.

\section{MDS Challenges}

In each of the four cycles of CIM we indentify real and potential obstacles that will hinder or at least slow down the industrial implementation of MDS. These obstacles are translated into challenges for each cycle which will to be addressed in the following (see Fig. 12):

\section{Challenges in Science}

To attain a pure product it is essential to understand the interaction particle-flow in mixing, segregation and splitting 
into the different density fractions. This can be achieved by creating scientific models to simulate and predict the behaviour of the particles and the magnetic fluid in an inhomogeneous magnetic field. In addition, new sensor technology must be developed for material identification before (feed acceptance and value estimation), during (process control through imaging) and after (product quality control) separation. Wetting parameters need to be studied to avoid compromising the separation, which is based on density. The search for polymer additives and recipes can achieve the highest possible quality of the products. Critical waste characteristics need to determine the value creation by MDS. This requires fundamental research.

\section{Challenges in Engineering}

Engineers from industry and researchers from the technical sciences must cooperate in the engineering cycle to develop full scale MDS installations. In particular, process liquid control and new quality control technology must be implemented to optimise the separation of PE and PP. An issue of importance for implementing MDS on an industrial scale is the prevention of particles blocking the system. Also, legal protection of the newly developed technologies must be sought.

\section{Challenges in Market Supply}

Product quality and specifications have to be developed in cooperation with early equipment manufacturers and early adopters in the recycling industry. After all, robust production and profitability are the first requirements from the business holders. New product-market combinations have to be identified. Collaboration with the industrial users of the reclaimed plastics is essential to enable the upgrade plastic mixtures to high-grade raw materials that can be used for the manufacturing of new high-quality products. Market research is necessary on the potential usefulness of the inevitable residual stream of low-grade material. This may spark new activity in the engineering cycle when new technology is needed to accomplish this.

\section{Challenges in Market Demand}

The demand for raw materials is growing day by day, both from the fully developed western societies as from the rapidly evolving economies of China and India. Increasing population and product consumption have created awareness in governments for the need to stimulate technology developers and raw material users for new technologies that enable an increased reuse of end-of-life products and thus reduce waste generation. If mechanical recycling proves to be the best option (as evidenced from Life Cycle Analyses) of the three alternatives for end-of-life plastics, then it is of paramount importance that MDS technology is readily available. Also, legislation must be approved and introduced to stimulate the recycling industry to adopt this most environmentally friendly alternative.

Finally, the high degree of self-organisation in the individual cycles requires a entrepreneurial body to monitor, interrelate, synchronize and coordinate the progress around the circle. We call this the challenge of the 'circle captain'.

The above innovation challenges are implemented in the European FP7 project: W2Plastics, high-purity products from plastic waste [23]. To reach this goal is a combined effort of both academic and industrial partners. The work is divided into 12 different work packages (Table 4). The interrelation between the work packages is plotted in Fig. (13).

Fig. (14) shows where in CIM the work packages are located. Because every cycle is involved in at least two work packages, the industrial implementation of MDS technology qualifies as a true four star innovation. Central to the processes - taking place in parallel in the four cycles - is the

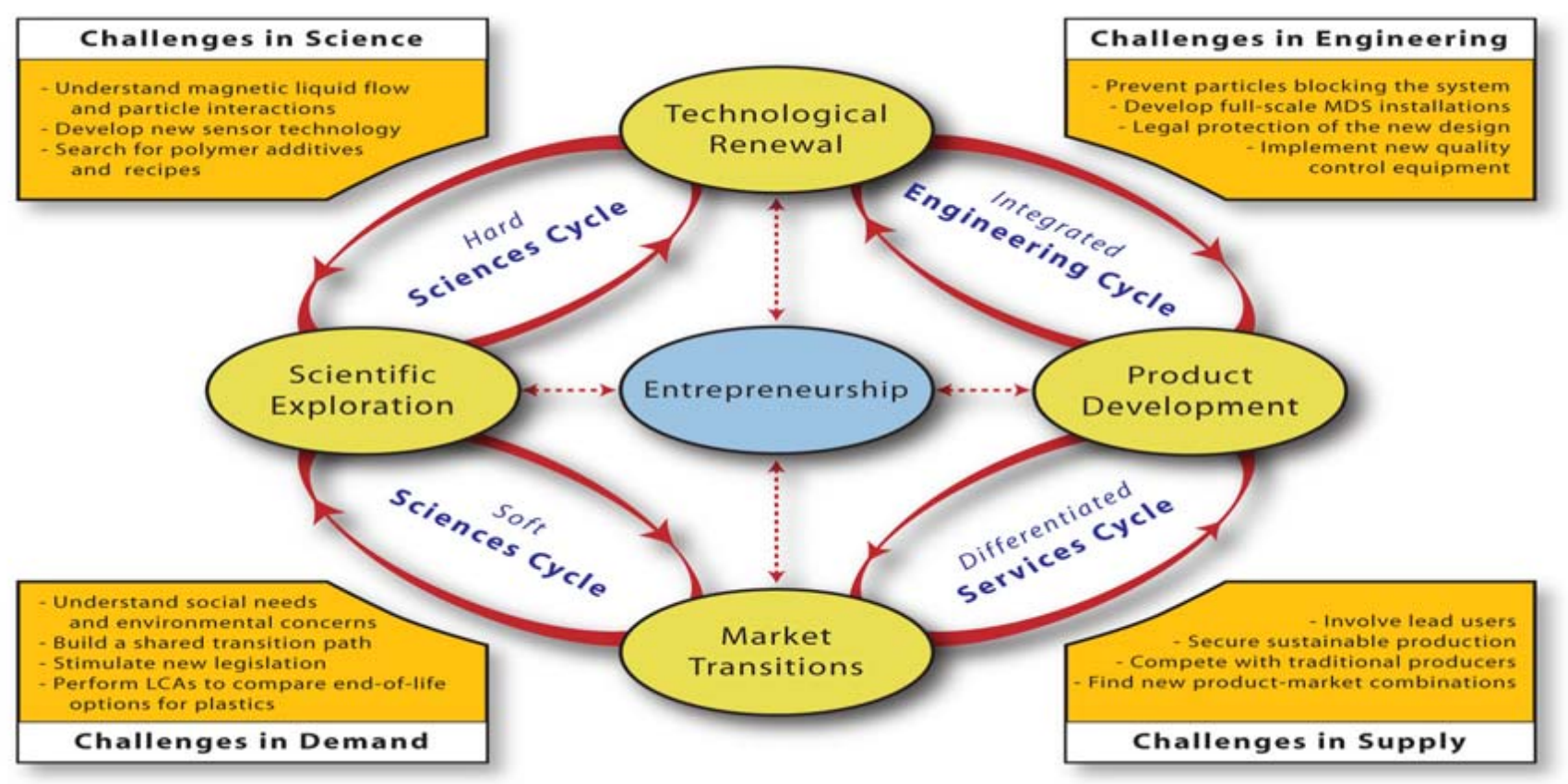

Fig. (12). The Cyclic Innovation Model for accelerating commercial use of MDS Technology 
entrepreneur or circle captain. Work packages 11 and 12 resort under his responsibility because they are connected with all four cycles.

Table 4. The Work Packages and their Description

\begin{tabular}{|c|l|}
\hline Work Package & \multicolumn{1}{c|}{ Description } \\
\hline \hline 1 & $\begin{array}{l}\text { Characterisation of plastics streams from different } \\
\text { sources and locations }\end{array}$ \\
\hline 2 & $\begin{array}{l}\text { Market analysis, tests and applications of high purity } \\
\text { secondary plastics }\end{array}$ \\
\hline 3 & Life Cycle Assessments \\
\hline 4 & Green solution for process residues \\
\hline 5 & $\begin{array}{l}\text { Chemical and physical improvement of the quality of } \\
\text { recycled polyolefins }\end{array}$ \\
\hline 6 & Numerical simulation and design of the MDS \\
\hline 7 & Development of the MDS \\
\hline 8 & Ultrasound system design \& data processing \\
\hline 9 & Sensors for control equipment and quality assessment \\
\hline 10 & Technology platform in industry \\
\hline 11 & Workshops, training and dissemination \\
\hline 12 & Project management \\
\hline
\end{tabular}

Finally, technology push refers to a community that may be excellent in designing and building technical functions (technical excellence), but still underperforms economically because of a communications barrier between the technicaland market-driven organisations (in terms of CIM: a barrier between the upper and lower part of Fig. 14). Both worlds make their own choices and plans; they "throw" their wishes and results more or less over the fence to the other side. The $20^{\text {th }}$ century can be characterized as a period of technology push. The overall control of work package 12 should prevent such a system error.

\section{CONCLUSIONS}

We present three main conclusions:

\section{Sustainability}

The ever increasing demand for raw materials is quickly exhausting the natural resources; we have excavated them from the earth for ages, in some cases as long ago as the Bronze Age. The only way we can sustain the use of many of these raw materials is to reuse them again and again. After products have reached their end-of-life phase, the raw materials from which they were made have to be recycled for reuse in the manufacturing of new products. We must attempt to close as many raw material cycles as possible. The incentive to do this should not be approached from a pure economic perspective. Alternatively, we should not be

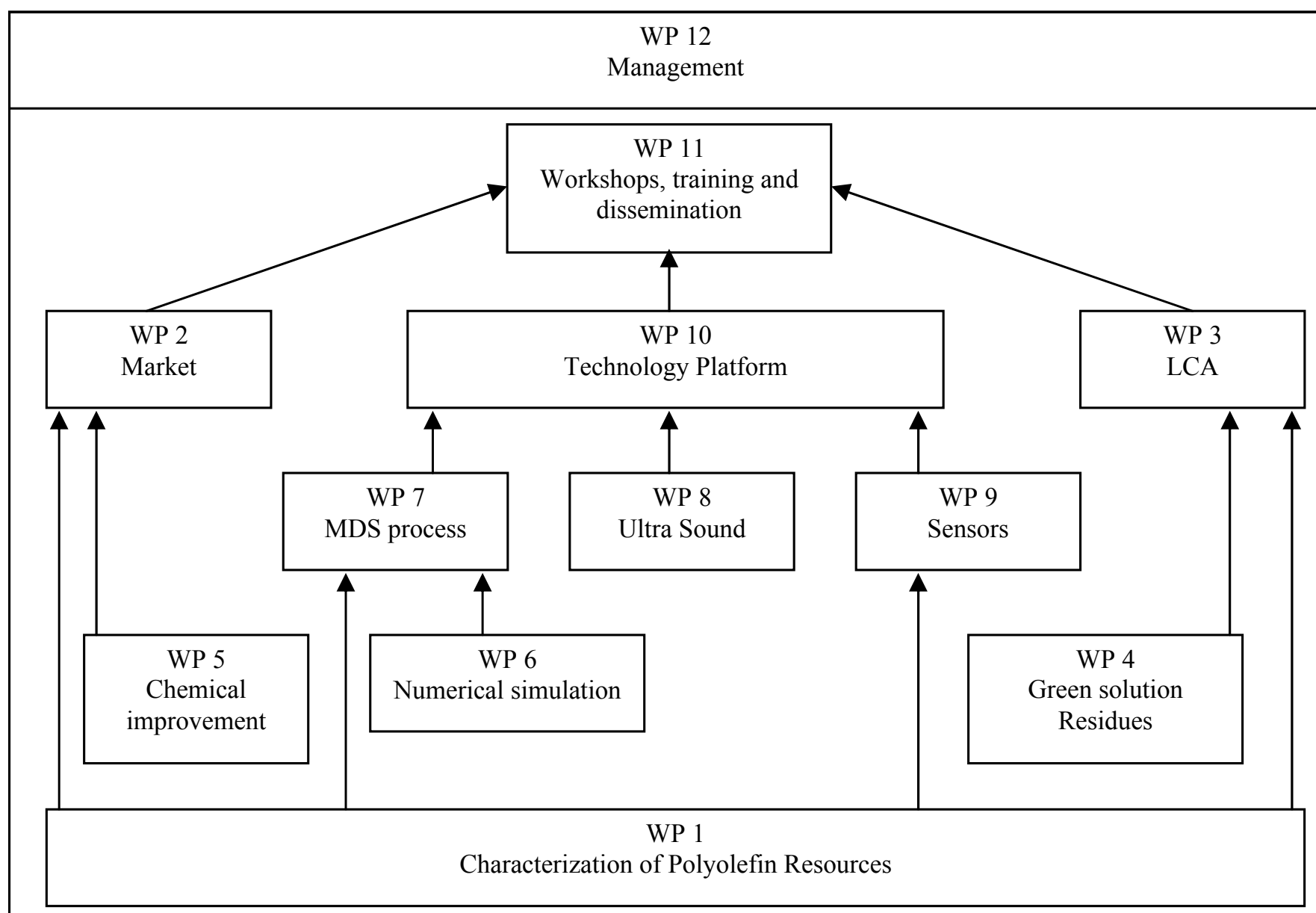

Fig. (13). Overview and hierarchy of the work packages of W2Plastics. 


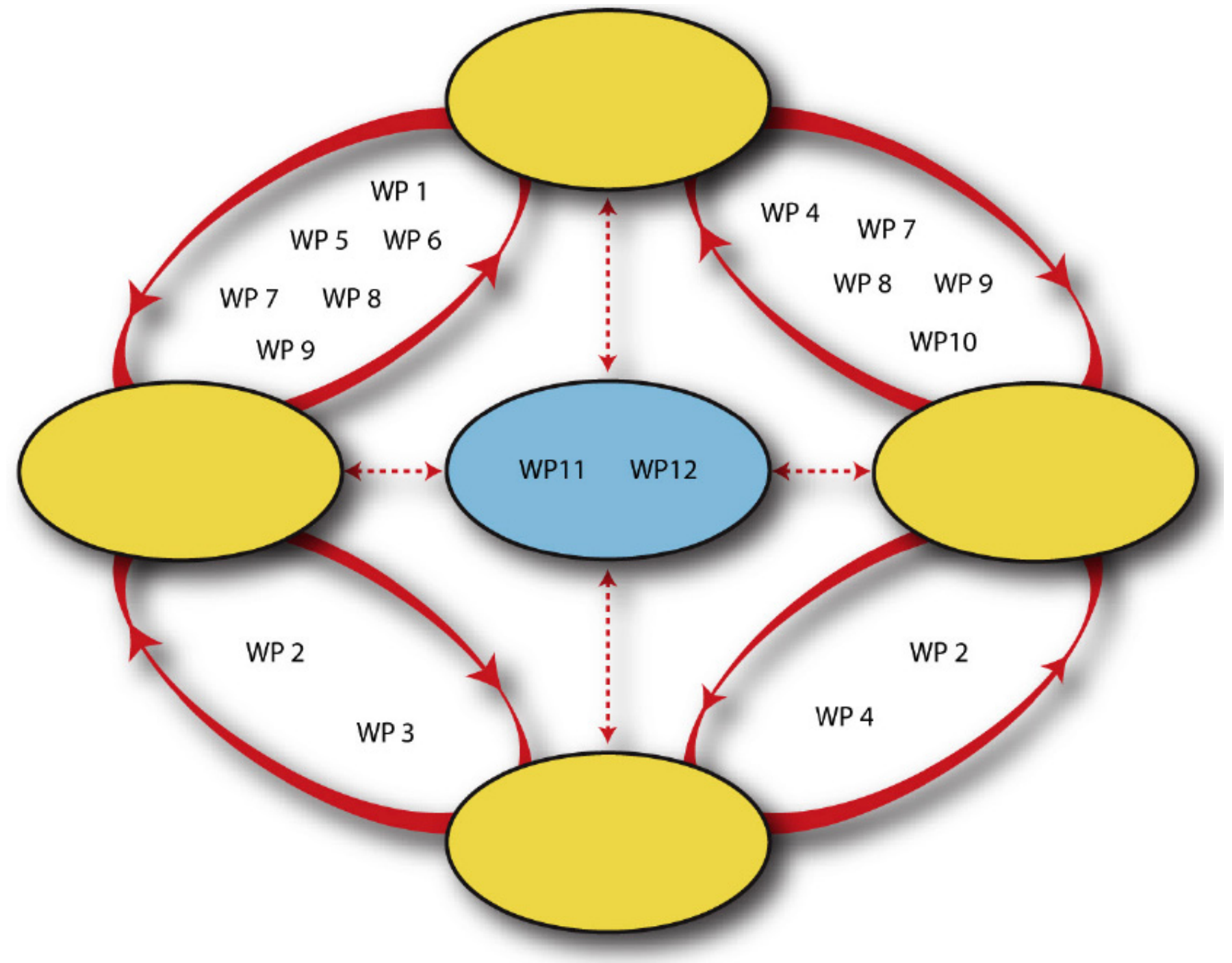

Fig. (14). The MDS work packages mapped onto the Cyclic Innovation Model.

motivated only by a sustainable philosophy that purports to recycle at all cost, even if that cost exceeds the gain (financially or ecologically). System studies (such as LCAs and other 'big picture' approaches) must be carried out to calculate the optimal approach towards recycling within the larger framework that encompasses all aspects of sustainability. Such studies may guide us in making the right choices in recycling.

\section{MDS}

Experiments with MDS technology were carried out on four different material streams: PET and contaminants, copper and aluminium, copper and precious metals, and PP and PE. The separation results were very encouraging for all four applications. The technology has a high accuracy like the sink-float process. Moreover, the control of the density is easy and there are no operational problems with liquid disposal. The MDS has the potential to simplify the PET recycling process significantly, and it can generate a better quality of the product. The new technology is more flexible in the size of scale with the separation of copper-aluminium. In addition, MDS is cheaper than the precious metals recovery from a heavy non-ferrous fraction. Our first experience with separating PP and PE looks technically, environmentally and commercially most promising.

\section{CIM}

The Cyclic Innovation Model (CIM) describes the four principal components in innovation and shows how they are interconnected. The activities in CIM are not part of a linear chain, but they involve cyclic processes in an eco-circle: innovations build on innovations. In addition, CIM emphasizes the hard and soft aspects of those processes; both aspects must be addressed to be successful. This is particularly true for the forthcoming transition period mankind is facing. In this transition to a sustainable future, technical ambitions are mixed with human emotions. CIM visualizes the many different innovation activities - in the fields of science, technology, products and markets - that need be planned, interrelated, synchronized, balanced and financed. This results in a transparent overview of the required resources and it helps to reveal the organisative and cultural boundaries that are potential candidates for significant delays (see Fig. 14 for the MDS project). Experience with CIM shows that key to innovation is the use of a shared mental framework, that allows more creative 
interaction between a large number of diverse players. From this perspective, CIM acts as an interdisciplinary communication model, connecting many diverse people with diverse roles within and across four different networks: hard scientists and environmental specialists, soft scientists and engineers, technological researchers and market experts. For complex innovation projects that aim at true sustainable solutions, such as MDS, a framework like CIM is indispensable.

\section{REFERENCES}

[1] W. McDonough and M. Braungart, Cradle to Cradle. Remaking the way we make things, North Point Press, 2002.

[2] L. H. Lovins, "Rethinking production", State World 2008, pp. 3840, 2008.

[3] B. Berkovski, Magnetic fluids and applications handbook, UNESCO series of learning materials, ISBN 1-56700-062-2, 1996.

[4] J. Svoboda, Magnetic Techniques for the Treatment of Materials, Kluwer Academic Publishers, ISBN 1-4020-2038-4, 2004.

[5] E. J. Bakker and P. C. Rem, "Magneto-hydrostatic separation of PET", Proceedings of the $5^{\text {th }}$ international conference for conveying and handling of particulate solids, Sorrento, Italy. Aug 27-31, 2006.

[6] L. Muchova, E. J. Bakker and P.C. Rem, "Precious Metals in Municipal Solid Waste Incineration Bottom Ash", Water Air Soil Pollut., vol. 9, pp. 107-116, January 2009.

[7] E. J. Bakker, P. C. Rem and N. Fraunholcz, "Upgrading mixed polyolefin waste with magnetic density separation", Waste Management, 29, pp. 1712-1717, 2009.

[8] H. Agterhuis, D. Trambitas, E.J. Bakker, P.C. Rem, "Comparing methods for reclaiming ferrofluids used in materials separation", Proceedings of the $22^{\text {nd }}$ international conference on solid waste technology and management, Philadelphia, PA, USA, March 1821, 2007.

[9] A. J. Berkhout, The Dynamic Role of Knowledge in Innovation. An Integrated Framework of Cyclic Networks for the Assessment of
Technological Change and Sustainable Growth, Delft University Press, Delft, 2000.

[10] J. E. Johansson, "Plastics - the Compelling Facts and Figures", Proceedings of the $6^{\text {th }}$ IdentiPlast biennial conference on the recycling and recovery of plastics, 2007.

[11] E. J. Bakker, P. C. Rem and N. Fraunholcz, "Novel method to separate polyolefins from sredder residue based on inverse magnetic density separation", Proceedings of the $6^{\text {th }}$ IdentiPlast biennial conference on the recycling and recovery of plastics, 2007.

[12] Delphi Consortium www.delphi.tudelft.nl

[13] H. W. Chesbrough, "Open innovation: the new imperative for creating and profiting from technology", Harvard Business School Press, Watertown, MA, USA, 2003.

[14] E.V. Hippel, "Democratizing Innovation", MIT Press, Boston, MA, USA, 2005.

[15] A. J. Berkhout, P. V. D. Duin, D. Hartmann, R. Ortt, "The cyclic nature of innovation: connecting hard sciences with soft values", Advances in the Study of Entrepreneurship, Innovation and Economic Growth, Elsevier, Amsterdam, vol. 17, 2007.

[16] R. Florida, Cities and the creative class, Routledge, New York, 2005.

[17] M. Donati, "Beyond synchronicity: The worldview of Carl Gustav Jung and Wolfgang Pauli”, J. Anal. Psychol., vol. 49, pp. 707-728, January 2004.

[18] C. Perez, Finance and technical change: A long-term view: Oxford University Press, pp. 1-18, 2002.

[19] R. E. Lucas, "On the Mechanics of Economic Development", $J$. Monetary Econ., vol. 22, pp. 3-42, January 1988.

[20] R. Kirschbaum, Open Innovation in Practice, Research-Technology Management, vol. 48, p. 24, 2005.

[21] J. Forrester, Industrial Dynamics, Productivity Press, Cambridge, 1961.

[22] P. M. Senge, The Fifth Discipline, the art and Practice of the Learning Organization. Doubleday, New York, 1994.

[23] P. C. Rem, S. Venga and F. Di Maio, "High-purity products from plastic waste: the W2plastics project", International Conference on Materials Science \& Engineering (BraMat), Brasov, 26-28, February 2009.

(C) Bakker et al.; Licensee Bentham Open.

This is an open access article licensed under the terms of the Creative Commons Attribution Non-Commercial License (http://creativecommons.org/licenses/by$\mathrm{nc} / 3.0 /$ ) which permits unrestricted, non-commercial use, distribution and reproduction in any medium, provided the work is properly cited. 